\title{
Alain Pagès, Zola et le groupe de Médan. Histoire d'un cercle littéraire
}

Damiano De Pieri

\section{(2) OpenEdition}

1 Journals

\section{Edizione digitale}

URL: http://journals.openedition.org/studifrancesi/553

DOI: 10.4000/studifrancesi.553

ISSN: 2427-5856

\section{Editore}

Rosenberg \& Sellier

\section{Edizione cartacea}

Data di pubblicazione: 1 aprile 2015

Paginazione: 180

ISSN: 0039-2944

\section{Notizia bibliografica digitale}

Damiano De Pieri, «Alain Pagès, Zola et le groupe de Médan. Histoire d'un cercle littéraire », Studi Francesi [Online], 175 (LIX | I) | 2015, online dal 01 avril 2015, consultato il 18 septembre 2020. URL : http:// journals.openedition.org/studifrancesi/553; DOI : https://doi.org/10.4000/studifrancesi.553

Questo documento è stato generato automaticamente il 18 settembre 2020.

\section{(c) (i) (9)}

Studi Francesi è distribuita con Licenza Creative Commons Attribuzione - Non commerciale - Non opere derivate 4.0 Internazionale. 


\title{
Alain Pagès, Zola et le groupe de Médan. Histoire d'un cercle littéraire
}

\author{
Damiano De Pieri
}

\section{NOTIZIA}

ALAIN PAGÈs, Zola et le groupe de Médan. Histoire d'un cercle littéraire, Paris, Perrin, 2014, pp. 479.

1 Sebbene la bibliografia critica e storico-letteraria dedicata al Naturalismo e ai suoi protagonisti sia ormai ampia e dettagliata, mancava una storia di sintesi del cosiddetto “groupe de Médan". Ed è proprio questo vuoto che Alain Pagès, direttore dei «Cahiers naturalistes», uno dei massimi esperti del Naturalismo e di Émile Zola, cui ha dedicato gran parte delle sue ricerche, ha voluto colmare con il presente volume.

2 Nei sei capitoli, inquadrati da un prologo (pp. 7-14) che presenta l'argomento e un epilogo (pp. 423-427) che ne trae le conclusioni, l'A. ripercorre cronologicamente la nascita, l'affermazione e la disgregazione del Naturalismo osservandolo attraverso la lente dell'esperienza collettiva, dei legami personali ed affettivi, ponendo al centro di questa rete di relazioni, ovviamente, la figura di Zola. Fin dalle prime pagine infatti («Le berceau provençal (1840-1868)», pp. 15-59) viene sottolineato lo spirito di amicizia e di gruppo del giovane Zola che dalla provincia del sud si ritrova a Parigi per muovere $i$ suoi primi passi da scrittore entrando a far parte, come è d'obbligo, delle cerchie letterarie animate dai maestri Flaubert e Goncourt, e diventando ben presto a sua volta un riferimento per scrittori poco più giovani di lui («Maîtres et disciples (1868-1876)», pp. 61-108). Ma è nei capitoli centrali («La bataille de L'Assommoir (1876-1878)», pp. 109-169 e «Le cercle de Médan (1879-1881)», pp. 171-267) che l'A. ricostruisce, in parallelo al consolidarsi dell'estetica naturalista, la nascita e l'affermarsi del gruppo composto, oltre che dallo stesso Zola, da Paul Alexis, Henry Céard, Léon Hennique, Joris-Karl Huysmans e Guy de Maupassant, consacrato letterariamente, com'è noto, dalla pubblicazione nel 1880 della raccolta Les Soirées de Médan. Questo cenacolo, 
definito da Alain Pagès un «foyer nécessaire pour que l'amitié se développe et que des projets intellectuels prennent corps» e un luogo "pour l'invention d'une nouvelle forme de littérature», diventa di conseguenza il simbolo del Naturalismo stesso, protagonista reale della battaglia intrapresa da Zola e dai suoi discepoli per il rinnovamento della letteratura. Infine, l'A. mostra come l'emergere di una nuova generazione e di posizioni letterarie diverse in seno al gruppo («La nouvelle vague naturaliste (1882-1887)», pp. 269-358) segni definitivamente i rapporti personali di Zola - che muteranno radicalmente con il concorso dell'«affaire Dreyfus»-, determini un superamento dell'estetica naturalista e porti allo scioglimento del gruppo di Médan che acquisirà nel tempo, attraverso le celebrazioni di Zola e del Naturalismo, una dimensione quasi leggendaria («L'avenir d'un mythe (1887-1930)», pp. 359-421).

3 Come dimostrato dalla bibliografia finale, il corpus è costituito dalla corrispondenza tra i vari protagonisti, le reciproche memorie, nonché le cronache e le rubriche letterarie dell'epoca. Nonostante la documentazione sia vasta ed eterogenea, Alain Pagès riesce ad orchestrarla con sapienza alleggerendo l'estremo rigore della ricostruzione storicoletteraria grazie a una scrittura narrativa che non esita a rievocare anche aneddoti apparentemente insignificanti per completare un mosaico in cui vita e letteratura si fondono restituendo così un ritratto completo dei protagonisti. 